ANNALES

POLONICI MATHEMATICI

$87(2005)$

\title{
Bi-Lipschitz trivialization of the distance function to a stratum of a stratification
}

\author{
by AdAm PARUsiński (Angers)
}

\begin{abstract}
Given a Lipschitz stratification $\mathcal{X}$ that additionally satisfies condition $(\delta)$ of Bekka-Trotman (for instance any Lipschitz stratification of a subanalytic set), we show that for every stratum $N$ of $\mathcal{X}$ the distance function to $N$ is locally bi-Lipschitz trivial along $N$. The trivialization is obtained by integration of a Lipschitz vector field.
\end{abstract}

The existence of Lipschitz stratifications of complex analytic or real subanalytic sets ([5], [7], [8]) allows one to trivialize these sets locally along each stratum so that the trivialization is bi-Lipschitz. In this paper we show the following refinement of this result that answers positively a question posed to us by M. Ferrarotti and E. Fortuna.

TheOREM 1. Let $\mathcal{X}$ be a Lipschitz stratification of a locally closed subset $X \subset \mathbb{R}^{n}$ and let $N$ be a stratum of $\mathcal{X}$. Suppose additionally that $\mathcal{X}$ satisfies condition $(\delta)$ of Bekka-Trotman along $N$. Then $\mathcal{X}$ can be trivialized locally along $N$ in such a way that the distance to $N$ is preserved by the trivialization and the trivialization is bi-Lipschitz.

We explain in Remark 2 below what we precisely mean by bi-Lipschitz trivialization.

We always assume that the strata of $\mathcal{X}$ are $C^{2}$ subvarieties of $\mathbb{R}^{n}$. We say that $\mathcal{X}$ satisfies condition $(\delta)$ of Bekka-Trotman along $N$ (see [1]) if for any $p \in N$, there are $c_{0}>0$ and $\varepsilon>0$ such that for all $q \in X$ satisfying $\operatorname{dist}(p, q)<\varepsilon$, there is a unit vector $\mathbf{v} \in T_{q} S$, where $S$ is the stratum containing $q$, such that

$$
d \varrho(\mathbf{v}) \geq c_{0},
$$

2000 Mathematics Subject Classification: Primary 32S15, 32S60; Secondary 32B20.

Key words and phrases: Lipschitz stratification, Whitney stratification, BekkaTrotman regularity condition, distance function, subanalytic sets.

This research was partially supported by the European project RAAG HPRN-CT00271. 
where $\varrho$ denotes the distance function to $N$. Geometrically it means that the strata of $\mathcal{X}$ transversally intersect the levels of $\varrho$ with the angle uniformly bounded away from zero. We note (cf. [5, Proposition 7.1]) that each Lipschitz stratification satisfies the Kuo-Verdier condition (w) (cf. [9]), and hence any Lipschitz stratification of a subanalytic set is Whitney regular. Whitney's (b)-regularity trivially implies condition $(\delta)$ of Bekka-Trotman.

One may ask whether any subanalytic function $f$ on $X$ can be trivialized along strata of a stratification of $X$ so that the trivialization is bi-Lipschitz. For this general question the answer is definitely negative, even for families of analytic function germs of two complex or real variables (see [2], [3]). Besides the distance to a stratum and similar distance-like functions (see Remark 7 below), it is not clear to us for what other types of functions the answer to this question is positive.

The proof of Theorem 1 is fairly elementary and uses the method introduced in [5]. The author thanks T. Mostowski for interesting discussions concerning the problem.

Proof of Theorem 1. Fix a stratum $N$ and $p \in N$. Let $k=\operatorname{dim} N$. Denote by $X^{i}$ the union of strata of dimension $\leq i$ and by $d_{i}(q)$ the distance from $q$ to $X^{i}$. The open ball centred at $q$ and of radius $r$ will be denoted by $B(q, r)$. As above, the distance function to $N$ will be denoted by $\varrho$. We shall work in a small neighbourhood $U$ of $p$ in $X$ that we will make smaller if necessary. All vector fields will be tangent to strata.

We will be sometimes sloppy about constants. In general, $\varepsilon>0$ will be used to denote very small constants, $M$ very large positive constants, $C>0$ will denote a universal constant and $L>0$ a universal constant for the Lipschitz condition. Our main tool will be the extension property of Lipschitz vector fields (cf. [7, Remark 1.3], [8, Proposition 1.3], [5, Proposition 1.1] and [7, Lemma 1.7]).

The main idea of the proof is simple. Given a system of Lipschitz vector fields $\mathbf{e}_{1}, \ldots, \mathbf{e}_{k}$ defined in a neighbourhood of $p$ in $N$ that form a basis of $T_{p} N$. By Gram-Schmidt orthonormalization we may suppose that for each $p^{\prime}$ close to $p, \mathbf{e}_{1}\left(p^{\prime}\right), \ldots, \mathbf{e}_{k}\left(p^{\prime}\right)$ is an orthonormal basis of $T_{p^{\prime}} N$. We will show that there exist extensions of $\mathbf{e}_{1}, \ldots, \mathbf{e}_{k}$ to Lipschitz vector fields $\widehat{\mathbf{e}}_{1}, \ldots, \widehat{\mathbf{e}}_{k}$, defined on $U$, that satisfy

$$
d \varrho\left(\widehat{\mathbf{e}}_{i}\right) \equiv 0 \quad \text { for } i=1, \ldots, k .
$$

Let $\pi: U \rightarrow N$ denote the closest point projection. Using the argument of Proposition 1.1 of [5] or Lemma 1.7 of [7], we may then change the vector fields $\widehat{\mathbf{e}}_{i}$ so that they additionally satisfy

$$
d \pi\left(\widehat{\mathbf{e}}_{i}\right)=\mathbf{e}_{i}, \quad i=1, \ldots, k .
$$

Then the theorem follows by integration of these vector fields as in loc. cit. 
REMARK 2. By local bi-Lipschitz triviality of $\varrho$ along $N$ we mean the following. Let $p_{0} \in N$ and let $U$ be a small neighbourhood of $p_{0}$ in $X$. Define $U_{N}=U \cap N$ and $U_{p}=\pi^{-1}(p) \cap U$ for $p \in U_{N}$. Let $\varrho_{p}$ be the restriction of $\varrho$ to $U_{p}$. Then for every $p_{0}$ there is such a $U$ and a bi-Lipschitz homeomorphism $\Phi: U_{p_{0}} \times U_{N} \rightarrow U$ such that $\pi(\Phi(x, y))=y$ and $\varrho(\Phi(x, y))=\varrho_{p}(x)$.

By adding the open stratum $\mathbb{R}^{n} \backslash X$ we may, moreover, assume that the above trivialization is the restriction to $X$ of a local bi-Lipschitz trivialization of the ambient space $\mathbb{R}^{n}$.

Now we present the details.

LEMma 3. There are a neighbourhood $U$ of $p$ and positive constants $\varepsilon, c, C, L$ such that for any $q_{0} \in U \backslash N$ there is a Lipschitz vector field $\mathbf{v}$ defined on $B\left(q_{0}, \varepsilon \varrho\left(q_{0}\right)\right)$, with Lipschitz constant $L \varrho\left(q_{0}\right)^{-1}$, such that for all $q \in B\left(q_{0}, r\right)$,

$$
\begin{gathered}
\|\mathbf{v}(q)\| \leq C, \\
d \varrho(\mathbf{v}(q)) \geq c .
\end{gathered}
$$

Proof. First fix an arbitrary small neighbourhood $U$ of $p$ in $\mathbb{R}^{n}$. It will be replaced later by a smaller neighbourhood of $p$. Let $q_{0} \in U \backslash N, S$ the stratum containing $q_{0}$, and let $\mathbf{v}_{0} \in T_{q_{0}} S$ be a vector satisfying condition (1). The vector field $\mathbf{v}$ of the statement of the lemma will satisfy $\mathbf{v}\left(q_{0}\right)=\mathbf{v}_{0}$.

Assume now that $q_{0} \in X^{j} \backslash X^{j-1}$. We abbreviate $\varrho\left(q_{0}\right)$ by $\varrho_{0}$. The proof is by induction on $j=k+1, \ldots, \operatorname{dim} X$. First we explain the inductive step. Let $\varepsilon^{\prime}, c^{\prime}, C^{\prime}, L^{\prime}$ be the constants for which the lemma holds for each $q_{0} \in X^{j-1} \cap U$. Fix a large $M>0$, in particular we require $M^{-1} \leq \varepsilon^{\prime} / 3$.

CASE 1: Suppose that for all $i=k, \ldots, j-2$,

$$
d_{i}\left(q_{0}\right) \leq M d_{i+1}\left(q_{0}\right) \text {. }
$$

Then $\varrho_{0} \leq M^{n-k} d_{j-1}\left(q_{0}\right)$. The vector field on $X^{j-1} \cup\left\{q_{0}\right\}$ that is identically equal to zero on $X^{j-1}$ and to $\mathbf{v}_{0}$ at $q_{0}$ is Lipschitz with Lipschitz constant $\varrho_{0}^{-1} M^{n-k}$. By [7] and [8] this vector field can be extended to a Lipschitz vector field on $X$ with constant $\varrho_{0}^{-1} M^{n-k} L_{\mathcal{S}}$, where $L_{\mathcal{S}}$ denotes a universal constant depending only on the Lipschitz stratification. An easy computation shows that this extension has the required property on $B\left(q_{0}, \varepsilon \varrho_{0}\right)$, provided $\varepsilon>0$ is chosen sufficiently small $\left(\varepsilon \leq \frac{1}{2}\left(M^{n-k} L_{\mathcal{S}}\right)^{-1} c_{0}\right.$ would do, where $c_{0}$ is given by Bekka-Trotman's condition).

Let $k^{\prime}>k$ be the minimum dimension of strata $S$ such that $p \in \bar{S} \backslash S$. Note that if $j=k^{\prime}$ then we are necessarily in Case 1 . Thus Case 1 gives also the initial step of the induction.

CASE 2: Set $j_{0}=i_{0}+1$, where $i_{0}$ is the largest $i \in\{k, \ldots, j-2\}$ for which (4) fails. That is, (4) holds for $i \geq j_{0}$ but

$$
d_{j_{0}-1}\left(q_{0}\right)>M d_{j_{0}}\left(q_{0}\right) .
$$


Fix $q^{\prime} \in X^{j_{0}} \backslash X^{j_{0}-1}$ such that $\left\|q_{0}-q^{\prime}\right\|=d_{j_{0}}\left(q_{0}\right)$. We have

$$
\left\|q_{0}-q^{\prime}\right\|=d_{j_{0}}\left(q_{0}\right)<M^{-1} d_{j_{0}-1}\left(q_{0}\right) \leq M^{-1} \varrho_{0} .
$$

Thus if $M^{-1} \leq \varepsilon^{\prime} / 3$ then $B\left(q_{0}, \varepsilon^{\prime} \varrho_{0} / 3\right) \subset B\left(q^{\prime}, \varepsilon^{\prime} \varrho\left(q^{\prime}\right)\right)$ and the vector field constructed for $q^{\prime}$ works as well for $q_{0}$.

Next we construct the desired vector fields $\widehat{\mathbf{e}}_{i}$ locally near each $q_{0} \in$ $U \backslash N$. Let $\widetilde{\mathbf{e}}_{i}$ be arbitrary Lipschitz extensions of $\mathbf{e}_{i}$ onto $U$. Define, on $B\left(q_{0}, \varepsilon \varrho\left(q_{0}\right)\right)$,

$$
\widehat{\mathbf{e}}_{i}:=\widetilde{\mathbf{e}}_{i}-\frac{d \varrho\left(\widetilde{\mathbf{e}}_{i}\right)}{d \varrho(\mathbf{v})} \mathbf{v}
$$

where $\mathbf{v}$ is given by Lemma 3. Clearly $d \varrho\left(\widehat{\mathbf{e}}_{i}\right) \equiv 0$. We show that the $\widehat{\mathbf{e}}_{i}$ are Lipschitz extensions of $\mathbf{e}_{i}$. We use the following obvious lemma.

Lemma 4. Let $f_{1}, f_{2}$ be two bounded Lipschitz functions with Lipschitz constants $L_{1}, L_{2}$ and bounded by $C_{1}, C_{2}$ respectively. Then the product $f_{1} f_{2}$ is Lipschitz with constant $C_{1} L_{2}+L_{1} C_{2}$. If moreover $\left|f_{1}\right| \geq c_{1}$ then $1 / f_{1}$ is Lipschitz with constant $L_{1} c_{1}^{-2}$.

To use the lemma we need to establish the Lipschitz constants and the bounds for the vector fields $\mathbf{v}, d \varrho(\mathbf{v}), d \varrho(\widetilde{\mathbf{e}})$.

For $\mathbf{v}$ they are given in Lemma 3 . Since $\varrho$ is a distance function, $|d \varrho(\mathbf{v})| \leq$ $\|\mathbf{v}\| \leq C$. Moreover, the second order partial derivatives of $\varrho$ can be universally bounded by a multiple of $\varrho^{-1}$. (If $N$ is a $C^{2}$ submanifold then $\varrho^{2}$ is of class $C^{2}$ in a neighbourhood of $p$.) Consequently, by Lemma 4, the Lipschitz constant of $d \varrho(\mathbf{v})$ can be universally bounded by a multiple of $\varrho\left(q_{0}\right)^{-1}$.

Finally,

$$
d \varrho(q)\left(\widetilde{\mathbf{e}}_{i}(q)\right)=d \varrho(q)\left(\widetilde{\mathbf{e}}_{i}(q)-\mathbf{e}_{i}(\pi(q))\right)+d \varrho(q)\left(\mathbf{e}_{i}(\pi(q))\right) .
$$

Since $\pi$ is the projection to the closest point, the last summand on the right hand side is equal to zero. On the other hand, $\left\|\widetilde{\mathbf{e}}_{i}(q)-\mathbf{e}_{i}(\pi(q))\right\| \leq$ $L \varrho(q)$, where $L$ denotes the Lipschitz constant of $\widetilde{\mathbf{e}}_{i}$. Therefore $d \varrho(q)\left(\widetilde{\mathbf{e}}_{i}(q)\right)$ is bounded by $L \varrho(q)$ and is Lipschitz (with a universal Lipschitz constant). This concludes the argument showing that the vector fields $\widehat{\mathbf{e}}_{i}$ of (5) are Lipschitz.

Note also that $\widehat{\mathbf{e}}_{i}$, extended by $\mathbf{e}_{i}$ to $N \cup B\left(q_{0}, \varepsilon \varrho\left(q_{0}\right)\right)$, is Lipschitz. We use the following lemma to glue the Lipschitz vector fields thus constructed.

Lemma 5 (after Lemma 3.1 of [4]). Given $\alpha>0$. There is $M>0$ and an (infinite) family of functions $\varphi_{m} \geq 0$ on $U \backslash N$ such that

(1) for each $x \in U \backslash N$ only finitely many $\varphi_{m}(x) \neq 0$,

(2) $\sum_{m} \varphi_{m} \equiv 1$,

(3) for all $m \in \mathbb{N}$, diam $\operatorname{supp} \varphi_{m} \leq \alpha \operatorname{dist}\left(\operatorname{supp} \varphi_{m}, N\right)$,

(4) each $\varphi_{m}$ is Lipschitz with constant $M\left(\operatorname{dist}\left(\operatorname{supp} \varphi_{m}, N\right)\right)^{-1}$. 
Thus choosing $q_{m}$ so that $\operatorname{supp} \varphi_{m} \subset B\left(q_{m}, \varepsilon \varrho\left(q_{m}\right)\right)$ and choosing all constants appropriately we construct

$$
\widehat{\mathbf{e}}_{i}=\sum \varphi_{m} \widehat{\mathbf{e}}_{i, m}
$$

where $\widehat{\mathbf{e}}_{i, m}$ denote the vector fields constructed above in $B\left(q_{m}, \varepsilon \varrho\left(q_{m}\right)\right)$. These vector fields have the desired properties. This ends the proof of Theorem 1.

Note that in Lemma 3 we may additionally require that $d \pi(\mathbf{v}) \equiv 0$. Indeed, we may write

$$
d \pi(\mathbf{v}(q))=\sum_{i} \lambda_{i}(q) \mathbf{e}_{i}(\pi(q)) .
$$

The functions $\lambda_{i}$ thus defined are Lipschitz with a universal constant. Then

$$
\widehat{\mathbf{v}}=\mathbf{v}-\sum_{i} \lambda_{i} \widehat{\mathbf{e}}_{i}
$$

has the desired properties.

COROLlary 6. There exists a Lipschitz vector field $\mathbf{w}$ defined on $U \backslash N$ such that $d \pi(\mathbf{w}) \equiv 0$ and for all $q \in U \backslash N$,

$$
\|\mathbf{w}(q)\| \leq C \varrho(q), \quad d \varrho(\mathbf{w}(q)) \geq c \varrho(q) .
$$

Proof. Locally on $B\left(q_{0}, \varepsilon \varrho_{0}\right)$ we put $\mathbf{w}:=\varrho \widehat{\mathbf{v}}$. Then we glue these vector fields using Lemma 5 as above.

REMARK 7 . The above argument allows us to trivialize along $N$ functions more general than the distance function to the stratum $N$.

More precisely, suppose $\mathcal{X}$ is a Lipschitz stratification of $X \subset \mathbb{R}^{n}$ such that Whitney's condition (b) is satisfied along a stratum $N$. Let $U$ be an open subset of $X$ and let $f: U \rightarrow \mathbb{R}$. We suppose that for every $p \in U \cap N$ there is a neighbourhood $V$ of $p$ in $\mathbb{R}^{n}$ and a $C^{2}$ function $\varphi: V \rightarrow \mathbb{R}^{n-k}$ such that $f=\|\varphi\|$ on $V \cap X, 0$ is a regular value of $\varphi$, and $N=\varphi^{-1}(0)$. Then $f$ is locally bi-Lipschitz trivial along $U \cap N$.

\section{References}

[1] K. Bekka et D. Trotman, Propriétés métriques de familles $\Phi$-radiales de sous-variétés différentiables, C. R. Acad. Sci. Paris Sér. I 305 (1987), 389-392.

[2] J.-P. Henry and A. Parusiński, Existence of moduli for bi-Lipschitz equivalence of analytic functions, Compositio Math. 136 (2003), 217-235.

[3] -, - Invariants of bi-Lipschitz equivalence of real analytic functions, in: Geometric Singularity Theory, Banach Center Publ. 65, Inst. Math., Polish Acad. Sci., Warszawa, 2004, 67-75.

[4] B. Malgrange, Ideals of Differentiable Functions, Oxford Univ. Press, 1966.

[5] T. Mostowski, Lipschitz equisingularity, Dissertationes Math. 243 (1985). 
[6] T. Mostowski, Lipschitz stratifications and Lipschitz isotopies, in: Geometric Singularity Theory, H. Hironaka et al. (eds.), Banach Center Publ. 65, Inst. Math., Polish Acad. Sci., Warszawa, 2004, 179-210.

[7] A. Parusiński, Lipschitz stratification. A review of results, in: Global Analysis in Modern Mathematics, K. Uhlenbeck (ed.), Proc. Symposium in Honor of Richard Palais' Sixtieth Birthday, Publish or Perish, Houston, 1993, 73-91.

[8] —, Lipschitz stratifications of subanalytic sets, Ann. Sci. École Norm. Sup. 27 (1994), 661-696.

[9] J.-L. Verdier, Stratifications de Whitney et théorème de Bertini-Sard, Invent. Math. 36 (1976), 295-312.

Département de Mathématiques

UMR $6093 \mathrm{du}$ CNRS

Université d'Angers

2, bd Lavoisier

49045 Angers Cedex 01, France

E-mail: Adam.Parusinski@univ-angers.fr

Reçu par la Rédaction le 28.11.2004

Révisé le 18.8.2005 